\title{
The Use of Line Matching Discriminant Power Line Extraction In Aerial Images
}

\author{
Jing Liu \\ Department of Electrical Engineering, North China Electric Power University (Baoding), Baoding, \\ 071000, China
}

email: 2065478882@qq.com

Keywords: Hough transform; Matching degree; extraction of power line; straight line matching discriminant method

\begin{abstract}
This paper studies the extraction of power line in aerial image based on the Hough transform method. In the detection of Hough transform, it is easy to be interfered by the noise, which leads to false detection. Considering the characteristic of power line in aerial images, we propose a set of threshold based on Hough transform line detection results in a straight line matching discriminant method. This new method can effectively remove the false detection of power lines, and improve the accuracy of power line detection.
\end{abstract}

\section{Introduction}

In the field of computer vision, line detection is a very important subject. It is a very important task for the identification of other elements or objects. In the aspect of the extraction of aerial image of power line, the accuracy of line detection is a very important task. The Hough transform algorithm has good stability, and has a certain anti-interference ability in line detection, which has disadvantage is that the amount of computation and storage space is too large.

In succession, an adaptive Hough transform and a random Hough transform are proposed to reduce the computation; The best Kaider window function is proposed to improve the anti-interference ability and the detection precision. In the research of power line extraction, the recognition and extraction of power lines is a key technology. The complexity and the diversity of the changes of the background make the power line extraction more difficult.

In the reference paper[1], the Ratio operator is used to extract the pixels of the power line, and then extract and connect to each segment by using the method of Ratio transform, and the power line is extracted in the tracking connection power line fault section;

The method of maximum entropy thresholding based on genetic algorithm is introduced, and the method of image segmentation based on genetic algorithm is introduced, and the method of communicating area is used to extract and recognize the target in the connected region of [2].

These methods have initially solved the extraction of power lines.

\section{Characteristics of power line}

When UAV (Unmanned-Aerial-Vehicle) is performing the power line inspection task, the general situation of it is usually inclined present 15 to 45 degrees above the power line, which shows a certain regularity. (See in reference [3])

After we analyze the basic properties of high voltage power line and the distribution characteristics of the high voltage power line in aerial images. The followings are what we summarized the main characteristics of the aerial images in power line which are referred to the SAR road extraction of road features [4] on the basis of the analysis:

First, due to the restricted to the height of the aerial, the power line in the aerial image display are very small pixels, and long lines, basically throughout the image if it is under no fault.

Second, because of the characteristics of transmission lines erected, the power lines topology structure in the aerial image is simple, and lines are often parallel between each other, basically with 
no intersection

Also, Power line background is natural and artificial landscape, may be very complex, such as roads, rivers, buildings, etc. The edges of these objects in the image are so similar to power lines that cause some difficulties for power line detection.

According to these characteristic, we conclude such features: power lines are parallel straight line which is throughout the whole photo, and the width of it is very small (about 7 pixels according to the experience of aerial height). (See [5])

\section{A brief introduction to the standard Hough transform algorithm[6]}

Hough transform is a parameter estimation technique of "voting". The principle is the use of the point - line duality between the image space and the introduction of the parameter space. By simple accumulation in the parameter space, and then to detecting line in the Hough parameter space accumulator peak. In essence, it is the clustering of image space points.

The oblique section equation of a straight line: $y=k x+b$; the same line have the same slope and intercept. In the parameter space, the polar coordinates are expressed as: $\rho=x \cos (\theta)+y \sin (\theta)$

We assume that every point is constant, so parameter space becomes a function of $(\rho, \theta)$, and every straight line has the same $(\rho, \theta)$.

$\theta$ changes from 0 to 180 degrees of compensation can be set according to needs, will be (x,y) corresponding to the cumulative array. We count the number of points that have the same $(\rho, \theta)$, followed by the sort to give a maximum of data points $(\rho, \theta)$ and corresponding data point was the point on a line. Reflected in the parameter space, as most of the intersection curve $(\rho, \theta)$ corresponding to the pixel points.

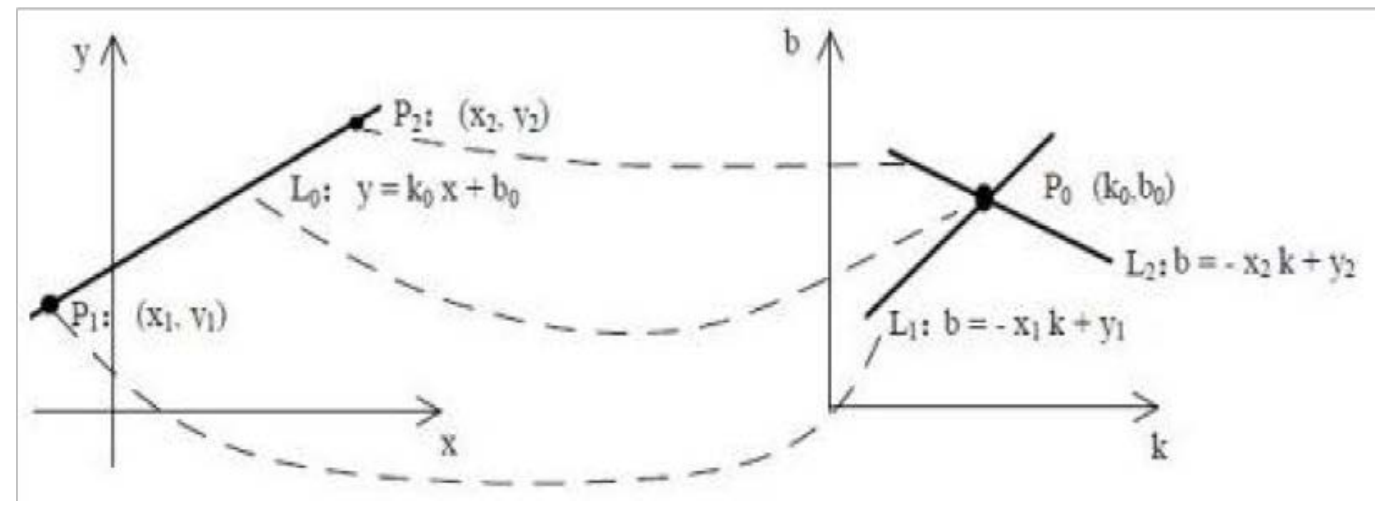

Fig.1.Hough transformation diagram

Hough transform is a cumulative process point by point, the standard Hough transform has good fault tolerance and robustness. But it generated a lot of miscellaneous data in the local traversal process, while maintaining the accuracy of the calculation and performance also have higher requirements.

With the rapid increase in computer performance, this question is not too important, the paper focus on improving the accuracy of the power line extracted on the basis of this.

\section{The method of detecting straight line with setting the threshold value}

Firstly, according to the characteristics of the data stored in the MATLAB digital image, the following coordinate system is established (The position of each pixel is positive integer) 


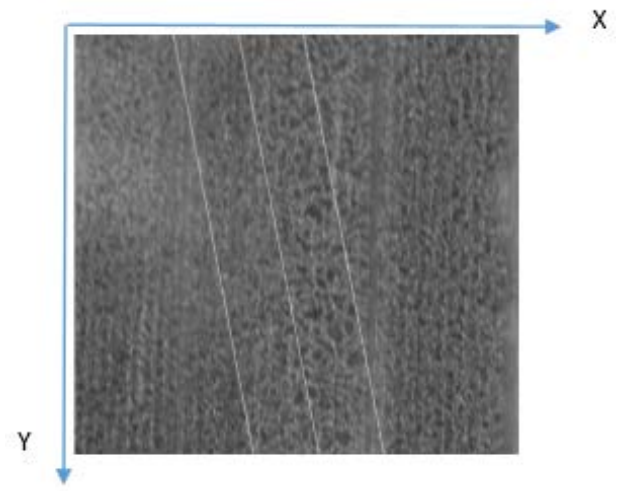

Fig.2.A right angle coordinate system based on digital image

By analyzing the characteristics of linear display in aerial images, we found that the linear pixel distribution of different slopes is different. The establishment of the following coordinates on the image, the pixels on the line is along the $|\mathrm{x} 1-\mathrm{x} 2|$, |y1 - y2| spread direction.

When the $|\mathrm{x} 1-\mathrm{x} 2|>|\mathrm{y} 1-\mathrm{y} 2|$, the pixels are stretched along the $\mathrm{X}$ axis, and the ideal line takes approximately the average interval:

Interval $=\left[\frac{|x 1-x 2|}{\left|y 1-y_{2}\right|}\right]+1$

When the $\mid \mathrm{x} 1$ - $\mathrm{x} 2|-| \mathrm{y} 1$ - $\mathrm{y} 2 \mid$, the pixels are stretched along the Y axis, and the ideal line takes approximately the average interval:

Interval $=\left[\frac{|y 1-y 2|}{|x 1-x 2|}\right]+1$

Since Unmanned Aerial Helicopter Flight Characteristics of regularity, general situation of unmanned aerial vehicle (UAV) in power line above the ramp between 15 to 45 degrees angle of shot. We can use it as Constraint conditions of linear structure. According to the UAV's Shooting condition setting, we just ignore this condition (Horizontal and vertical lines).and we get 12 kinds of power line in the aerial image.

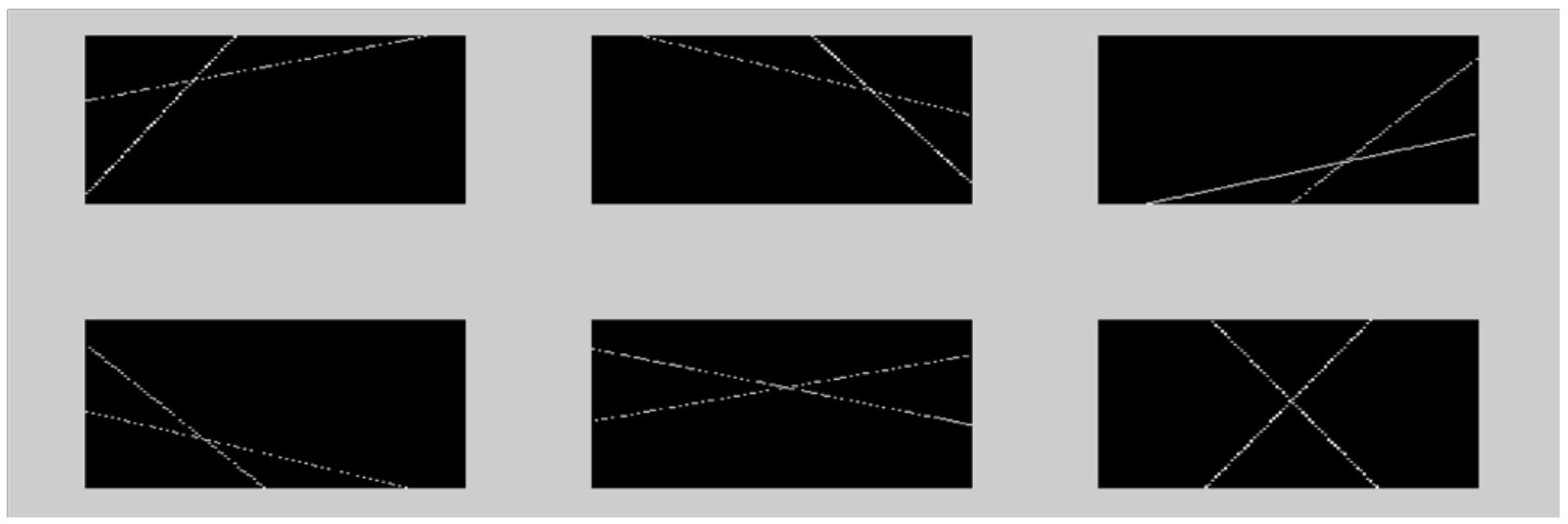

Fig.3.12 kinds of lines in aerial image

Considering that the point of the edge detection of the power line is not uniformly distributed in this line, the ideal line will be improved. In the direction of the pixel spread, we allow an Interval floating, and have a local pixel matching. The number of pixels that match the number of pixels will be a part of the structure to improve the line.

As shown in Figure five: (X0, Y0) is a point in transformed line after the Hough transform. 


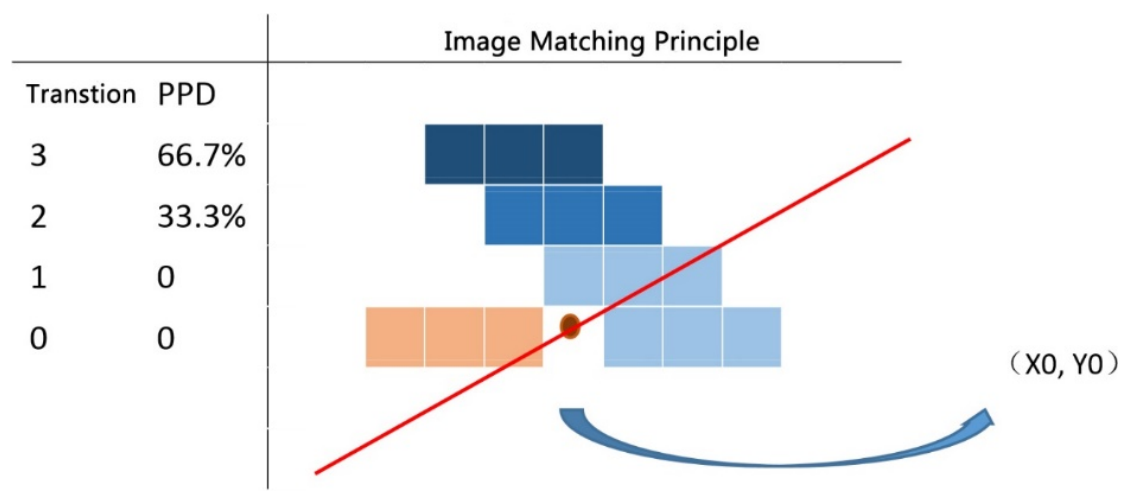

Fig.4.Image matching principle

Test the matching degree between original image and structure line

\author{
Matching degree $\quad$ PPD $=\frac{\text { Pixel_origin }}{\text { Pixel_total }}$ \\ Pixel_total:Total number of pixels in the line for the construction of 1 points \\ Pixel_origin:The number of pixels that have the same position with the Structure line in original \\ PPD:the matching degree for calculation \\ When PPD>th (th refers to threshold), the line we tested can be power line. On the contrary, it is
} image not.

\title{
Concluding remarks
}

Based on The test results by Hough transform, combined with the characteristics of power line in three in aerial images:

Power lines are parallel line, throughout the entire image with very small pixel width, we conclude the method of detecting straight line with setting the threshold value. At the same time, through the imaging characteristics of the lines in the detailed analysis of aerial digital image to construct displayed in digital image of 12 kinds of basic forms of linear, combined with aerial images improved ideal straight line is constructed, and image matching the best straight line was obtained.

After the test, in an image with complex background, under the setting of the threshold, we detected Hough transform's error-detection of power lines effectively. Finally, we achieved that power lines in aerial image can be extracted accurately.

\section{References}

[1] LiChaoYang, YanGuangJian, XiaoZhiQiang, LiXiaoWen, GuoJun, WangJinDi. Automatic Extraction of Power Lines from Aerial Images [J]. Chinese Journal graphics, 2007, 06:1041-1047.

[2] HuangXiaoNing,ZhangLiangZhen. A Method to Extract Insulator Image from Aerial Image of

[3] ZhuFangFang,GuHongBing,SunJing. An improved Hough transform line detection algorithm [J]. Computer technology and development, 2009, 05:19-22.

[4] TongWeiGuo. Study on identification and transmission line state detection method based on aerial image [D]. North China Electric Power University, 2011.

[5] CaiKe. Research on the specific target detection technology of helicopter aerial image [D]. Nanjing Aerospace School, 2009.DOI:10.7666/d.y1811623.

[6] LiuLiang. Research and engineering application of power line tracing algorithm for transmission line inspection of transmission line [D]. Shandong University, 2013 